Nat. Acad. Sci., 68, 1274 ; 1971) have now made bilayers with phospholipids, in which the spin label is covalently attached at various points on the chain. The paramagnetic resonance signals from oriented sheets of such bilayers can then give the average orientation of the label group with respect to the plane of the bilayer, within the time scale of the resonance relaxation time. Thus, if the interchange between possible geometrical states occurs more rapidly than $10^{8}$ times per second the observed orientation will be the timeaveraged direction, which is simply perpendicular to the bilayer plane for a label with its vector along the paraffin chain. If it is slower, the method will give an average orientation in space rather than time. Geometrical states of such long persistence in a paraffin would imply a high degree of cooperativity of motion, with a large cluster of chains swinging together into a new orientation, because the time scale of torsional motion about single bonds in isolated chains is of course much too fast to be of great interest.

Orientation is in fact observed: a label placed next to the polar head group at the outer part of the bilayer shows that this part of the paraffin chain is inclined at $60^{\circ}$ to the bilayer plane. Moving the label away from the head group, the apparent tilt diminishes, and near the methyl terminus at the centre of the bilayer, the chain is perpendicular to the plane. The tilt near the head group necessarily changes the packing density, and by an amount very similar to the difference between the density of a liquid and a solid paraffin. The middle of the sandwich then is in effect a liquid, but at the edges it behaves essentially as a layer of solid domains. McFarland and McConnell point out the mechanism that this structure suggests for cooperative processes: an amphiphilic molecule (paraffin-seeking at one end, polar at the other) thrust into such a bilayer will cause a local disturbance, which could bring about what they term a domino-effect, successive clusters of chains swinging from one bent state to another. By changing their tilt direction the paraffin chains can, moreover, create a cavity to receive such an amphiphile. The possible implications of this effect for transport and depolarization processes are numerous.

A related approach, which attempts to gather microscopic information about the properties of a paraffin cluster is that of Weber and his colleagues (Shinitzky et al., Biochemistry, 10, $2106 ; 1971)$. The rotational relaxation times of a molecule are related to the viscosity of its environment, and can, as all the world knows, be studied by measuring the depolarization of fluorescence. Shinitzky et al. have chosen to study first the nature of the paraffinic centre of a micelle. They use aromatic molecules of appropriate excited state lifetimes, which are insoluble in water, and calibrate the viscosity dependence of their fluorescence depolarization with paraffin oils of known viscosity. These species are then introduced into micelles, and used to measure the internal viscosity. This turns out to be in the range expected for a liquid, though greater than that of a bulk liquid paraffin. The rotation is anisotropic, with widely different relaxation times for rotations in and out of the molecular plane. By reference to experiments in other media, it is shown that the anisotropy is inherent in the geometry of the fluorescent molecule, and that the paraffin of the micelle is wholly isotropic.

An interesting observation of Shinitzky et al. is that in mixed micelles of detergent with cetyl alcohol or cholesterol--which introduce additional hydrophilic groups into the surface- the microscopic viscosity in the interior is strikingly increased. This is in nice accord with the evidence on the effects of cholesterol on the structure of membrane bilayers.

\section{HOMING \\ The Mystery Deepens}

from our Animal Behaviour Correspondent THE fact that many pigeons are able to home rapidly and accurately when released considerable distances away from their lofts in unfamiliar territory means that they must be able to use some system of bicoordinate navigation, and cannot simply use landmarks. The basis for this remarkable navigational ability is, however, so far unknown. The only complete theory proposed to date is that they are able to obtain information from the Sun about their position relative to that of their home loft, a view

\title{
Precursors of Ribosomal RNA
}

ANY molecular biologist casting around for new fields to plough would be well advised to steer clear of the mechanism of ribosome biosynthesis in bacteria, for a glance at next Wednesday's Nature New Biology is enough to convince anybody that that particular plot is already heavily if not over cultivated. No less than four groups, a dozen workers in all, report essentially identical experiments which lead them to conclude that the 16S RNA of the small subunit of the Escherichia coli ribosome is produced by cleaving bases from both ends of a larger precursor RNA.

A vast accumulation of circumstantial evidence indicates that the 16S RNA of $E$. coli ribosomes is not a direct product of transcription, and earlier this year Dahlberg and Peacock isolated what seemed to be this precursor molecule which, from its electrophoretic mobility in polyacrylamide gels, they estimated is some 200 bases longer than the mature ribosomal RNA. To prove that this molecule is indeed a precursor of 16S RNA, Lowry and Dahlberg have compared fingerprints of the two molecules. These showed that the precursor is a minimum of ninety-four nucleotides longer than the mature product and contains fewer methylated bases. Moreover, the fingerprint patterns indicate that these additional bases occur at both $5^{\prime}$ and $3^{\prime}$ ends of the precursor. Lowry, collaborating with Nomura, has also apparently shown that ribosomes reconstructed with the precursor RNA are inactive, but immature ribosomal particles containing the precursor can be isolated from cells. Presumably therefore the maturation of the precursor is essential for ribosomal function, and at least some steps in the maturation occur in ribosomes which are only partially assembled.

Like Lowry and Dahlberg, Fellner and his collaborators, who for the past several years have been doggedly analysing the base sequence of mature $16 \mathrm{~S}$ ribosomal RNA, conclude that the precursor has additional bases at both ends and that it is submethylated. They, however, estimate their precursor to be only about forty bases longer than the mature RNA; it might therefore be an intermediate product of maturation. Brownlee and Cartwright find that their preparations of precursor consist of molecules more than fifty-five bases longer than the mature molecule and they point out that because the $5^{\prime}$ base of the precursor does not carry a triphosphate group it may well not be the initial product of transcription. Finally, the Paces and their colleagues say that their precursor is some 10 per cent larger than the mature RNA and that during its maturation sequences are cleaved from both ends.

That $16 \mathrm{~S}$ ribosomal RNA is produced by the cleavage of both ends of a larger precursor is plainly beyond dispute. The initial product of the transcription of the $16 \mathrm{~S}$ ribosomal RNA gene, however, remains to be identified and characterized, for the molecules studied by these four groups are probably all intermediates between the gene transcript and the mature RNA. 